\title{
TRATAMIENTO DE LA FIEBRE TIFOIDEA CON TERRAMICINA
}

\author{
Por los Dxes, OSCAR UNDURRAGA. ALFREDo RALMANN y JULlo MENEghello \\ Cátedras de Pediatría de los Profs. A. Baeza Goñi y J. Meneghello Riveros. \\ Hospital M. Atriarán.
}

Desde agosto de 1951 hemos tenido oportusidad de tratar con terramicina 15 casus de ficbre tifoidea y 2 de paratifus $A: y$, respectivamente.

Si bien nuestra experiencia en el tratamiento de la fiebre tifoidea con cloromicetina era satisfactoria ${ }^{3 t}$, nos pareció justificado realizar otros ensayos terapéuticos. En este sentido podía suponerse que, dado el amplio espectro microbiano de la terramicina, era posible controlar casos en que la cloromicetina se mostraba inefjcaz, prevenir las complicaciones y recaídas y obtener la eliminación de los portadores convalecientes.

La experiencia extranjera, aunque reducida, ponía de manifiesto la eficacia que la terramicina revelaba en ciertas ocasiones en el control de la fiebre tifoidea. Así, Reilly y Earle ${ }^{5}$ obtienen en 4 de 6 enfermos resultados satisfactorios, utilizando dosis globales fluctuantes entre 2 y $3 \mathrm{~g}$, administrando la droga en dosis promedia variable de 100 a $200 \mathrm{mg}$ por kilo, de acuerdo a la gravedad de cada caso. Posteriormente, Killough y Magiel. tratan 5 enfermos, en 3 de los cuales se normaliza la temperatura en un promedio de 4,5 días. Sayer y $\mathrm{cols}^{\mathrm{e}}$ trataron un caso de fiebre tifoidea con éxito, utilizando una dosis diaria de $4 \mathrm{~g}$; este paciente recae y un segundo tratamiento produce una respuesta excelente.

Material. - Se compone de 15 enfermos de fiebre tifoidea, uno de paratifus A y otro de paratifus B. La edad de estos enfermos fluctuó entre 3 y 12 años, correspondiendo la mayor frecuencia entre los 6 y 9 años. En relación con el sexo, 9 fueron hombres y 8 mujeres. En todos los casos se trató de una infección tifica comprobada, sea por el aislamiento del germen y/o la reacción de aglutinación y/o un hemograma más o menos característico. Todos los enfermos llevaban una evolución antes del tratamiento de más de 7 días y un caso fué tratado muy tardíamente, a los 30 días de enfermedad. El promedio de duración de la enfermedad antes del tratamiento fué de 14 días. En general, la mayoría de nuestros enfermos correspondieron a casos que pudimos calificar de mediana gravedad. Hubo 4 casos catalogados como graves y 2 leves.

Tratamiento. - Consistía en el suministro de la droga de preferencia por vía oral y en forma de cápsulas. La dosis fué de $100 \mathrm{mg}$ por kilo de peso diario, repartida cada 6 horas y el promedio usado fué de $24,63 \mathrm{~g}$, fluctuando entre 35,5 y $14,7 \mathrm{~g}$. La proiongación de la terapia fué de 12 días. En los casos que no respondieron al tratamiento osciló su duración entre 3 y 14 días. Los plazos cortos obedecieron a manifiesta intolerancia por la droga.

Resultados. - En 7 de nuestros pacientes apreciamos resultados satisfactorios (gráficos 1 y 2) y en los 10 restantes consideramos que el tratamiento no fué eficaz (gráficos 3 y 4). Valoramos los resultados por la caída de la temperatura y la mejoría del estado general. En los casos en que el tratamiento fué eficaz, la normalización đe la curva tér- 
mica se obtuvo en plazos que fluctuaron entre 3 y 8 días, en promedio 5,1 días. En 3 enfermos la caída de la temperatura se produjo al tercer día de injciado el tratamiento, mejorando el estado general en forma más o menos simultánea con la normalización de la curva térmica (grífico 1). Hubo un grupo de 4 pacientes (gráfico 2), en que también se obtuvo la normalización de la curva térmica, pero en plazos más prolongados, 7 a 8 días. Sólo en 4 de los 10 enfermos, en los cuales consideramos que fué donde se pudo apreciar en forma más manifiesta la intolerancia por la droga, que se trađujo por vómitos intensos.

La forma en que se produjo la caída de la temperatura en aquellos casos calificados como satisfactorios fué: en crisis, 1 ; crisis y persistencia de febrículas, 2; en lisis, 2, y lisis y persistencia de febrículas, 2. En los casos que consideramos como fracasos, hubo 2 que normalizaron tardíamente la temperatura en lisis.

Experiencia en el tratamiento de la fiebre tlfoidea con terramicina (17 casos).

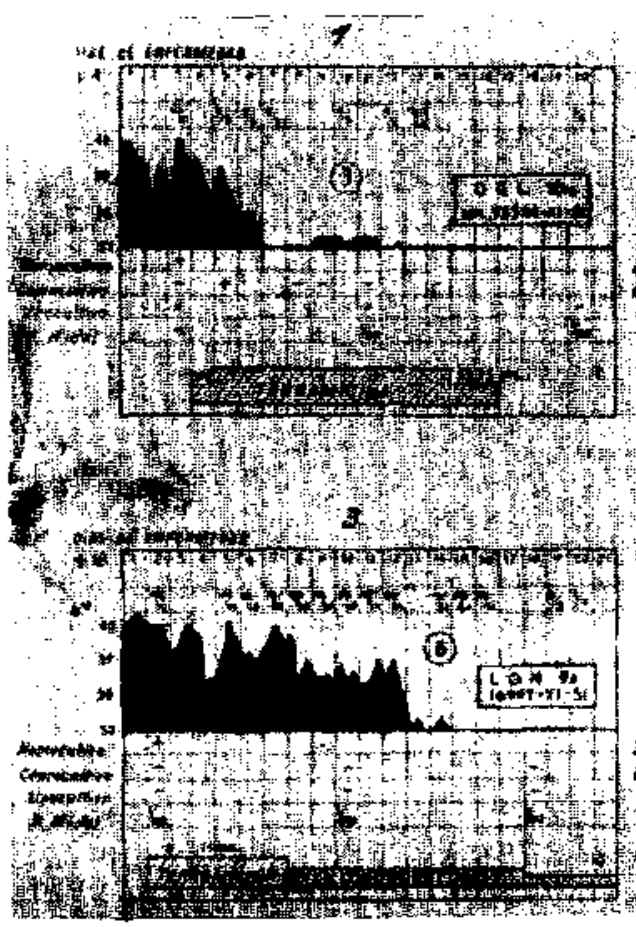

el tratamiento no produjo efectos, se pudo completar la terapia y la temperatura se normalizó tardíamente, como si la evolución hubiera sido espontánea (gráfico 4). En 6 casos la temperatura no descendió con la droga (gráfico 3), viéndonos obligados a suspenderla y a recurrir a la cloromicetina, con la que se obtuvo su normalización en 5 pacientes. En el 'caso restante fracasaron ambos medicamentos y la tifoidea hizo una evolución espontánea. En este grupo

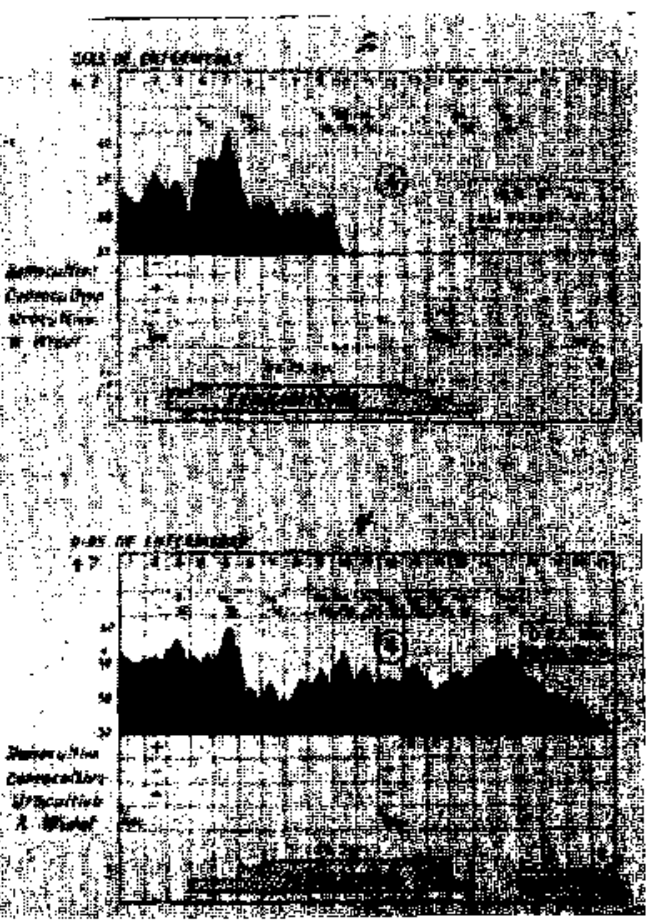

En cuanto al estado general, observamos su mejoria coincidiendo con la normalización de la temperatura.

La determinación de la sensibilidad del germen a la terramicina se verificó en 6 ocasiones, variando entre 4 y 16 microgramos por cc.

La terramicinemia la efectuamos en 9 de nuestros enfermos, encontrándose valores entre 3 y 10 microgramos por cc en aquellos casos que respondieron al tratamiento. En cambio, en 4 enfermos, 
en los que el tratamiento no surtió efecto, no se encontró el medicamento en la sangre.

Manifestaciones de intolerancia a la terramicina se observaron con frecuencia en nuestros pacientes, y por. este motivo debimos suspender la droga en 5 oportunidades. El síntoma mas destacado correspondió a vómitos muy intensos y repetidos, que sólo cedieron a la suspensión del medicamento. Otro signo muy llamativo en relación con el tratamiento fué la diarrea que se observó en 12 de nuestros 17 enfermos y que disminuia de intensidad, e incluso desaparecía, también con la suspensión del tratamiento.

\section{Resumen.}

Se relata la experiencia recogida en el tratamiento con terramicina de 17 enfermos con infección tífica confirmada y cuya enfermedad lievaba 7 ó más días de evolución.

La droga fué usada por vía oral en dosis de $100 \mathrm{mg}$ por kilo de peso diario, repartida cada 6 horas y por un plazo de 12 días. No se rebajó la dosis en relación con el descenso de la temperatura.

Los resultados apreciados por la normalización de la temperatura y la mejoría del estado general lo obtuvimos en 7 enfermos en plazos que variaron entre 3 y 7 días. En los 10 pacientes restantes consideramos que el tratamiento había fracasado, sea por intolerancia manifiesta a la droga traducida por vómitos persistentes e intensos, que obligaron a la suspensión de ella, o porque no se obtuvo la normalización de la temperatura, viéndonos obligados a usar cloromicetina, con lo que conseguimos el descenso de la temperatura.

No hubo diferencias en los resuitados obtenidos en relación con el sexo o edad de los pacientes.

No se observaron recaídas en el plazo de observación hospitalaria de nuestro material.

Aun cuando estimamos que nuestra experiencia es muy reducida para poder sacar conclusiones definitivas acerca de la eficacia de la terramicina en el tratamiento de las infecciones tíficas, pensamos que los resultados de este tratamiento son comparativamente inferiores a los obtenidos con la administración de cloromicetina en este grupo de enfermos.

\section{Summary.}

The authors report their experience with terramicine in 17 patients with typhoid fever of more than seven days duration. The drug was administered orally at a dose of $100 \mathrm{mg}$ per kilo per day, given every 6 hours for 12 days. In 7 patients the results, judged by the drop in temperature and the improvement in their general condition, were considered good. In the other 10 patients treatment failed due to intolerance to the drug manifested by persistent vomiting or due to the persistence of fever. The authors believe their results are comparatively inferior to those obtained with chloromycetin.

\section{Bibliografía.}

1.-KILLOUGH, J. H. and MAGILL, G. B. Clinical Observations on Terramycin in Epidemic Typhus, Amoeble Dysentery and Typhoid Fever. J. A. M. A. In Press.

2.-MENEGHELLO, J.; UNDURRAGA: O. y RAIMANN, A. - Cloramfenicol (cloromicetina) en el tratamiento de la fiebre tífoldea del niño. Rer. Ch. de Ped. 21 : 535,1950 .

3.-MENEGHELLO, J.; UNDURRAGA, O. y RUBIO, s. - Tratamlento de la fiebre thfoidea del niño con cloramfentcol suministrado cada 12 horas. Rev. $C h$. de Ped. 22: 25, 1951.

4.-MENEGHFLIO, J.; UNDURRAGA, O.; GALLO, A. y RATMANN, A. - Tratamiento de 100 casos de fiebre tifoldea en el niño, con clorarnfenicol (cloromicetina). Estudios comparativos con 159 enfermos que no rectbieron la droga. Rev. Méd. de Chile 79: 307,1951 .

5.-RFELLY, W. A. and EARLE, A. M. Treatment of Typhoid Fewer with Terramycin. J. Pediat. 38: 428-430, 1851.

6. -SAYHR, R.; MICHEL, J. C.; MOLI, F. C. and KIRBY, W. M. M. - Terramycin: Clinical, Pharmacologle and Bacterlologtc Studies. Am. J. Sc. 221: 256-263, 1951. 